\title{
Bilateral Total Knee Arthroplasty for Charcot Knees Associated with Tabes Dorsalis
}

\section{TO THE EDITOR}

In July 2011, a 71-year-old man presented for evaluation of his right knee, which had become swollen with no apparent cause. Clinical examination showed that the right knee was warm, swollen, and nonpainful with absent knee and ankle jerk reflexes. The range of motion of the right knee joint was slightly restricted. Radiographs of the knee showed bone destruction in the medial and anterior regions of the tibia. Despite this bone destruction, the patient reported no pain. Further questioning revealed a 20-year history of syphilis. He was admitted to our hospital for further investigation. In the hospital, a rapid plasma reagin test was positive. The cerebrospinal fluid total protein level was $830 \mathrm{mg} / \mathrm{L}$ (normal range, $150-450 \mathrm{mg} / \mathrm{L}$ ). The levels of rheumatoid factor and tumor markers were within their normal ranges. Multiple glucose measurements and testing for glycosylated hemoglobin showed normal results. No other significant laboratory findings were observed. Arthroscopy showed degenerative changes on the articular surface of the tibia. The anterior cruciate ligament and both menisci were intact, and there was marked synovial proliferation in the suprapatellar bursa. Arthroscopy exploration of sites of abnormal radiological findings revealed fragile fibrous and osseous tissues. Sufficient curettage of the lesion was performed. Histological examination showed nonspecific chronic synovitis without infectious findings or amyloid deposits in the synovium as well as degenerated and necrotic bone tissue without malignancy. The joint fluid showed no bacteria or crystals, and the fluid was bloody and transparent. Hence, the clinical diagnosis was neuropathic joint disease secondary to tertiary syphilis. A month after conservative therapy was begun, the limb swelling and clinical symptoms improved, and the patient was discharged from our hospital.

In October 2011, the patient presented to our department again. Clinical examination revealed slight local pain in the right knee; however, the knee had become misshapen with restricted movement. Radiographs of the right knee showed severe bone destruction of the lateral condyle of the femur and advanced bone destruction of the anterior tibia, and sclerosis was seen on the joint surface. To avoid a missed diagnosis, radiographs of the left knee were taken simultaneously; no obvious abnormities were found (Fig. 1a).

The patient did not tolerate casting because of necrobiosis lipoidica diabeticorum. In November, total knee arthroplasty (TKA) was performed using the Endo-Model $®$ rotational knee prosthesis (Waldemar LINK GmbH \& Co., Hamburg, Germany) by a medial parapatellar approach. The patient's knee was asymptomatic and fully functional for the next 2 years.

Two years after the first presentation, the patient unexpectedly presented to our hospital for the third time. He reported limited activity in the left knee, which was swollen but painless. Physical examination of the left knee revealed redness, swelling, and no obvious tenderness. Radiographs showed a narrowed joint space containing small bone chips. Because the damage was mild but the local inflammatory reaction was severe, surgery was not performed. The left knee was immobilized, and oral detumescence therapy was begun.

Two months later, the local symptoms had improved, but the deformity had become severe. Radiographs showed marked bone destruction within the tibia and distal femur and fine bone fragments within the knee joint (Fig. 1b). The patient requested TKA, which was performed in October 2013. The same rotational knee prosthesis used in the right knee was also used in the left knee. Fig. 2 shows the postoperative radiographs, which revealed successful implantation of the prosthesis.

Neuropathic arthropathy was described by Jean Martin Charcot as the progressive destruction of bone and soft tissue in patients with peripheral neuropathy. He noted the relationship between syphilis and severe arthropathy in 1868.

Evaluation of a patient with neuropathic arthropathy should include detailed history taking and a complete physical examination, including neurologic tests with special attention to pupillary abnormalities, sensory changes, and deep tendon reflexes. Other causes of neuropathic arthropathy must be ruled out. The diagnosis is also based on clinical findings such as a warm and swollen joint, and later examination may reveal joint dislocation, fractures, or deformities (1). Radiography is the preferred examination method. Especially in 


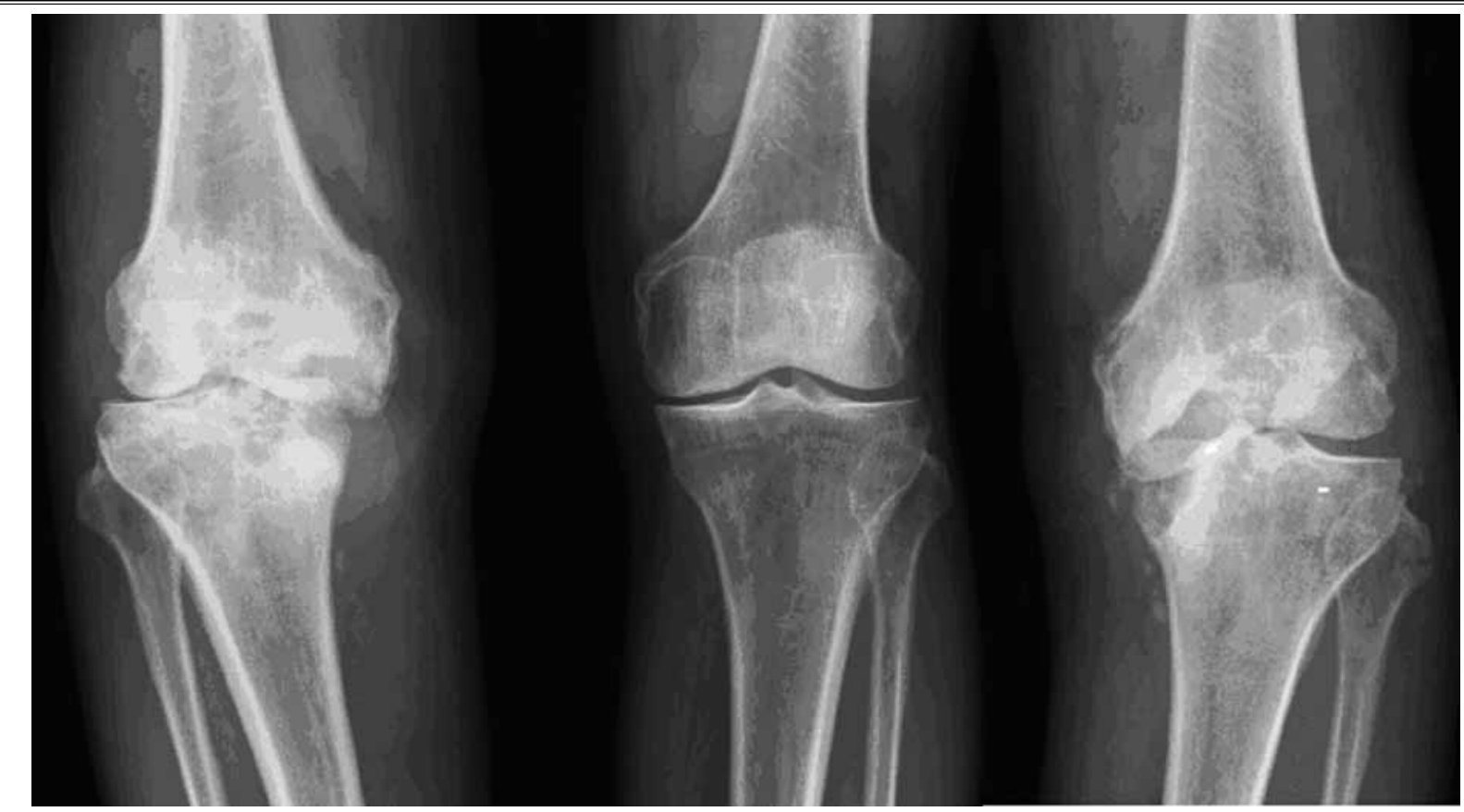

Fig. 1. Radiograph (a) of the right knee showing severe bone destruction of the lateral condyle of the femur, advanced bone destruction of the anterior tibia, and sclerosis on the joint surface. Radiograph (a) of the left knee showing no abnormalities. Radiograph (b) showing severe bone destruction within the tibia and distal femur and fine bone fragments within the joint.

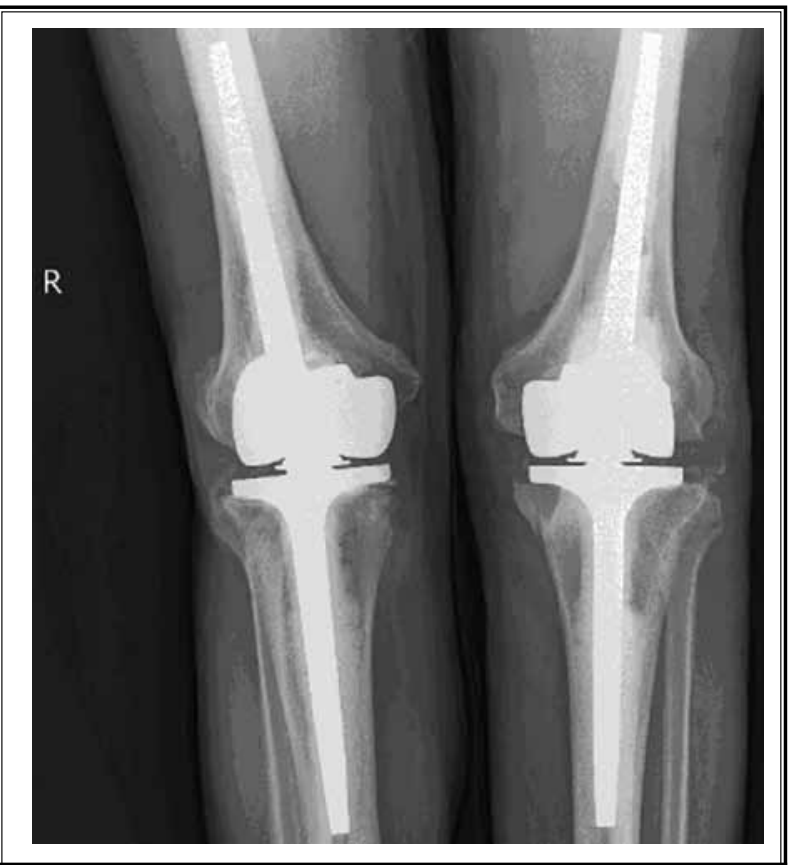

Fig. 2. Radiographs showing successful knee implantation. the late stages, there is apparent rapid bone resorption with destruction of the articular surface, subchondral sclerosis, and osteophyte development. Joint subluxation and intra-articular loose bodies can be seen (2). Magnetic resonance imaging and radionuclide scanning findings are less specific than radiographic findings $(3,4)$. Laboratory examination is mandatory and must include a complete blood count, determination of the glucose level, identification of glycated hemoglobin and rheumatoid factors, and treponemal and nontreponemal testing. The diagnostic gold standard for a Charcot joint is the histological appearance of fragments of bone and cartilage embedded within the synovium $(1,5)$.

Successful treatment of Charcot knee depends on an early diagnosis before the onset of limb-threatening deformity (6). When the knee joint is affected, conservative treatment alone is insufficient; immediate offloading and total encasement in a contact are necessary (7). TKA has been considered to be an absolute contraindication for this diagnosis because of the high incidence of serious complications $(2,8)$. In recent years, however, some authors have shown satisfactory results 
after TKA in patients with Charcot arthropathy (9-11). TKA in such patients has a high incidence of serious complications due to significant bone loss, poor bone quality, and ligamentous laxity. A TKA revision system may be available in cases of prospective complications. However, TKA can restore joint activity in a short period of time, which is more readily accepted by patients.

This is the first report of bilateral TKA within a short period of time in a patient with neuropathic arthropathy of both knees associated with tabes dorsalis. Because this case report involves a single patient, it may be more appropriate to speak about instructive remarks than to make definitive conclusions. When the knees are affected, conservative treatment alone is generally insufficient. TKA should not be considered a contraindication. Therefore, TKA revision systems must be available. However, the incidence of complications may be high. Notably, if one knee has Charcot knee, the other knee may also be affected within a short time.

\author{
Jin-Tao Liu, MD \\ Department of Orthopaedic Surgery \\ Suzhou Hospital of Traditional Chinese Medicine \\ Jiangsu, China \\ Longhua Hospital \\ Shanghai University of Traditional Chinese \\ Medicine \\ Shanghai, China \\ Xiao-Feng Li, MD \\ Longhua Hospital \\ Shanghai University of Traditional Chinese Medicine \\ Shanghai, China \\ Kun-Lin Xu, MD \\ Department of Orthopaedic Surgery \\ Suzhou Hospital of Traditional Chinese Medicine \\ Jiangsu, China
}

\author{
Zhi-Gang Zhang, MD \\ Department of Orthopaedic Surgery \\ Suzhou Hospital of Traditional Chinese Medicine \\ Jiangsu, China \\ Qi-Han Ma, MD \\ Department of Orthopaedic Surgery \\ Suzhou Hospital of Traditional Chinese Medicine \\ Jiangsu, China
}

Guan-Hong Liu, MD

Department of Orthopaedic Surgery

Suzhou Hospital of Traditional Chinese Medicine

Jiangsu, China

\author{
Zhen-Han Yu, MD \\ Department of Orthopaedic Surgery \\ Suzhou Hospital of Traditional Chinese \\ Medicine \\ Jiangsu, China \\ De-Zhi Tang, MD \\ Longhua Hospital \\ Shanghai University of Traditional Chinese \\ Medicine \\ Shanghai, China \\ E-mail: dztang702@126.com \\ Hong Jiang, MD \\ Department of Orthopaedic Surgery \\ Suzhou Hospital of Traditional Chinese Medicine \\ 889 Wuzhongxi Road \\ Suzhou, Jiangsu 215009, China. \\ E-mail: doctorhong@yeah.net
}




\section{References}

1. Babazadeh S, Stoney JD, Lim K, Choong PF. Arthroplasty of a Charcot knee. Orthopedic Reviews 2010; 2:e17.

2. Parvizi J, Marrs J, Morrey BF. Total knee arthroplasty for neuropathic (Charcot) joints. Clinical Orthopaedics and Related Research 2003; 416:145-150.

3. Dogan BE, Sahin G, Yagmurlu B, Erden I. Neuroarthropathy of the extremities: Magnetic resonance imaging features. Current Problems in Diagnostic Radiology 2003; 32:227-232.

4. Palestro CJ, Mehta HH, Patel M, Freeman SJ, Harrington WN, Tomas MB, Marwin SE. Marrow versus infection in the Charcot joint: Indium-111 leukocyte and technetium-99m sulfur colloid scintigraphy. Journal of Nuclear Medicine 1998; 39:346-350.

5. Sequeira W. The neuropathic joint. Clinical and Experimental Rheumatology 1994; 12:325-337.

6. Frykberg RG, Belczyk R. Epidemiology of the Charcot foot. Clinics in Podiatric Medicine and Surgery
2008; 25:17-28, v.

7. Nielson DL, Armstrong DG. The natural history of Charcot's neuroarthropathy. Clinics in Podiatric Medicine and Surgery 2008; 25:53-62, vi.

8. Vince KG, Cameron HU, Hungerford DS, Laskin RS, Ranawat CS, Scuderi GR. What would you do? Case challenges in knee surgery. The Journal of Arthroplasty 2005; 20:44-50.

9. Bae DK, Song SJ, Yoon KH, Noh JH. Long-term outcome of total knee arthroplasty in Charcot joint: A 10- to 22year follow-up. The Journal of Arthroplasty 2009; 24:11521156.

10. Tarukado K, Ikuta K, Fukutoku Y, Tono O, Doi T. Spontaneous regression of posterior epidural migrated lumbar disc fragments: Case series. Spine J 2013; S1529-S9430: 01265-5."

11. Troyer J, Levine BR. Proximal tibia reconstruction with a porous tantalum cone in a patient with Charcot arthropathy. Orthopedics 2009; 32:358. 